\title{
Therapeutic Internal Carotid Artery Occlusion for Large and Giant Aneurysms: A Single Center Cohort of 146 Patients
}

\author{
(D).S. Bechan, (D) C.B. Majoie, (D) M.E. Sprengers, DJ.P. Peluso, (D) M. Sluzewski, and (D)W.J. van Rooij
}

\begin{abstract}
BACKGROUND AND PURPOSE: At our institution, patients with large or giant ICA aneurysms are preferably treated with endovascular ICA balloon occlusion. Alternative treatment or conservative treatment is offered only for patients who cannot tolerate permanent ICA occlusion. In this observational study, we report the clinical and imaging results of ICA occlusion for aneurysms in a large single-center patient cohort.
\end{abstract}

MATERIALS AND METHODS: Between January 1995 and January 2015, occlusion of the ICA was considered in 146 patients with large or giant ICA aneurysms. Ninety-six patients (66\%) passed the angiographic test occlusion, and, in 88 of these 96 patients (92\%), the ICA was permanently occluded. In 11 of 88 patients with angiographic tolerance, ICA occlusion was performed with the patient under general anesthesia without clinical testing.

RESULTS: There was 1 hypoperfusion infarction after hypovolemic shock from a large retroperitoneal hematoma (complication rate $1.1 \%$ [95\% Cl, 1\%-6.8\%]). The mean imaging and clinical follow-up was 35 months (median 18 months; range, 3-180 months). On the latest MR imaging, 87 of 88 aneurysms (99\%) were completely occluded and 61 of 80 aneurysms (76\%) were decreased in size or completely obliterated. Of 62 patients who presented with cranial nerve dysfunction by mass effect of the aneurysm, 30 (48\%) were cured, 25 (40\%) improved, 6 (10\%) were unchanged, and 1 patient (2\%) was hemiplegic after a complication.

CONCLUSIONS: ICA occlusion for large and giant aneurysms after angiographic test occlusion was safe and effective. Two-thirds of eligible patients passed the angiographic test. Most aneurysms shrunk, and most cranial nerve dysfunctions were cured or improved.

L

arge and giant aneurysms of the internal carotid artery can be located intradurally from the ophthalmic segment upward or extradurally in the cavernous sinus. Intradural aneurysms may be symptomatic by SAH or decreased visual acuity by mass effect on the optic nerve or chiasm. Large and giant cavernous sinus aneurysms may be symptomatic by mass effect on cranial nerves IIIVII or by carotid cavernous fistula, epistaxis, or, rarely, SAH when ruptured. ${ }^{1-3}$

In general, treatment of large and giant ICA aneurysms is indicated after rupture to prevent recurrent SAH. Treatment is also indicated in symptomatic or asymptomatic unruptured intradural aneurysms to prevent first-time SAH or to alleviate symptoms of mass effect. Cavernous sinus aneurysms generally exhibit

Received May 5, 2015; accepted after revision June 8.

From Sint Elisabeth Ziekenhuis (R.S.B., J.P.P., M.S., W.J.v.R.), Tilburg, the Netherlands; and Academisch Medisch Centrum (C.B.M., M.E.S.), Amsterdam, the Netherlands.

Please address correspondence to W.J. van Rooij, PhD, Department of Radiology, Sint Elisabeth Ziekenhuis, Hilvarenbeekseweg 60, 5022GC Tilburg, the Netherlands; e-mail:wjjvanrooij@gmail.com

http://dx.doi.org/10.3174/ajnr.A4487 a benign clinical course. For both symptomatic and asymptomatic cavernous aneurysms, the risk profile of treatment should be balanced against the benign natural history.

Treatment of large and giant ICA aneurysms can be surgical, endovascular, or a combination. Surgery consists of direct clipping or bypass construction followed by parent ICA occlusion. ${ }^{4,5}$ During the past decades, endovascular techniques have largely replaced surgery for these aneurysms. Endovascular treatment can consist of ICA balloon occlusion, selective coiling with or without balloon or stent assistance, or parent ICA reconstruction with flow diverters. ${ }^{6-9}$

At the Sint Elisabeth Ziekenhuis, therapeutic ICA occlusion has been the preferred treatment for large and giant ICA aneurysms since 1995, despite the availability in the last decade of new endovascular devices, such as stents and flow diverters, intended to spare the parent ICA. We report the clinical and imaging results of ICA occlusion for aneurysms in a large single-center patient cohort. Although some data of our cohort have been published previously, in this article we intend to give a comprehensive and complete overview of our results with narrowed confidence intervals. 


\section{MATERIALS AND METHODS \\ General}

The indication for treatment of large and giant ICA aneurysms was discussed jointly with neurosurgeons and neurologists. Treatment was tailored to the individual patient by accounting for clinical presentation, aneurysm characteristics, patient age, comorbidity, and the patient's wishes. At our institution, patients with large or giant ICA aneurysms were preferably treated with endovascular ICA balloon occlusion. Selective coiling, flow-diverter treatment, bypass surgery, or conservative therapy was offered only for patients who could not tolerate permanent ICA occlusion. Large or giant ICA aneurysms in patients who presented with SAH were generally coiled first to prevent secondary hemorrhage, and, in a later stage, ICA occlusion was considered as definitive therapy. ICA occlusion in the acute phase of SAH was preferably not performed because vasospasm could induce ischemic events by decreasing reserve capacity after ICA occlusion.

\section{ICA Occlusion}

The protocol for therapeutic ICA occlusion has been described previously. ${ }^{10,11}$ In short, during ICA balloon test occlusion, angiography of the contralateral ICA and/or vertebral artery was performed to assess collateral flow via the anterior and posterior communicating arteries. Apart from clinical tolerance in awake patients, synchronous opacification of the cortical cerebral veins in the territories of the examined and occluded vessels was considered indicative of tolerance to permanent occlusion. We considered synchronicity if the relative delay in contrast filling of the cortical veins was $\leq 1$ second ( 2 frames in an angiographic run of 2 frames/second). This was best appreciated in forward and backward cine-loop with enhanced image contrast. After tolerance was determined, the ICA was permanently occluded proximal to the aneurysm with detachable balloons (Gold Valve no. 16 balloon; Nycomed, Paris, France, or Balt, Montmorency, France) or, in a short period when balloons were not available, with detachable coils. Aneurysm trapping was never performed. In the second half of the study period, therapeutic ICA occlusion was also performed in selected patients under general anesthesia without clinical testing. After permanent ICA occlusion, the patient was monitored for 24 hours in a medium care unit with special attention to blood pressure and fluid balance.

\section{Patients Without Tolerance for ICA Occlusion}

Patients who could not tolerate ICA occlusion were discussed again in a joint meeting with neurosurgeons and neurologists. Bypass surgery, selective coiling with or without stent assistance, or flow-diverter treatment was offered in symptomatic patients or asymptomatic patients with intradural aneurysms. In selected patients with asymptomatic or symptomatic cavernous sinus aneurysms, conservative treatment was recommended.

\section{Follow-Up Assessment}

After ICA occlusion, MR imaging was performed within 72 hours to evaluate thrombosis of the aneurysm and to detect (clinically silent) ischemic events in the watershed areas. In addition to T1and T2-weighted images, diffusion-weighted images were included in the protocol in later years. A clinical outpatient visit and

\begin{tabular}{lc} 
Location of 146 large and giant ICA aneurysms in 146 patients \\
\hline Extradural & $87(60 \%)$ \\
Cavernous segment & $86(59 \%)$ \\
Petrosal segment & $1(1 \%)$ \\
Intradural & $59(40 \%)$ \\
Hypophyseal segment & $15(10 \%)$ \\
Ophthalmic segment & $15(10 \%)$ \\
Posterior communicating artery & $4(3 \%)$ \\
Supraclinoid ICA dissection & $4(3 \%)$ \\
Supraclinoid other & $18(12 \%)$ \\
ICA bifurcation & $3(2 \%)$ \\
\hline
\end{tabular}

MR imaging were scheduled at 3 months and, in many patients, at various intervals thereafter. A substantial proportion of patients were the subject of several long-term MR imaging follow-up studies. $^{12-15}$

\section{Statistical Analysis}

Quantitative variables were expressed as mean (standard deviation), and categoric variables were expressed as frequencies or percentages. Statistical analysis was performed with MedCalc statistical software (version 14.12.0; MedCalc Software, Mariakerke, Belgium).

\section{RESULTS \\ Patients}

Between January 1995 and January 2015 occlusion of the ICA was considered in 146 patients with large or giant ICA aneurysms. There were 126 women (86\%) and 20 men (14\%), with a mean age of 57.8 years (median, 59 years; range, 16-91 years). The locations of the 146 ICA aneurysms are displayed in the Table. There were 87 extradural aneurysms (60\%) and 59 intradural aneurysms (40\%).

Clinical presentation was oculomotor dysfunction in 74 (50\%); SAH in 22 (15\%); decreased visual acuity in 19 (13\%); carotid cavernous fistula in $9(6 \%)$; hemiplegia in $2(1 \%)$; and trigeminal neuralgia, panhypopituitarism, and epistaxis each in 1 patient. In 17 patients (12\%), the aneurysm was an incidental finding.

\section{Patients with Tolerance for ICA Occlusion}

Of 146 patients who underwent ICA test occlusion, venous filling was synchronous in 96 (66\%). In 88 of these 96 patients (92\%), the ICA was permanently occluded. In 11 of 88 patients with angiographic tolerance, ICA occlusion was performed with the patient under general anesthesia without clinical testing. Six patients with SAH were treated with coiling first and were later treated with ICA occlusion for the same aneurysm. Of the remaining 8 aneurysms in 8 patients, 6 were selectively coiled, 1 was treated with a flow diverter, and 1 was left untreated. In 7 of these 8 patients ( 6 with ruptured cavernous sinus aneurysms and 1 with carotid cavernous fistula), ICA test occlusion was performed before parent vessel sparing treatment in order to be informed beforehand about tolerance and the possibility of carotid sacrifice as a potential bailout during treatment.

\section{Patients with Nontolerance for ICA Occlusion}

In 50 of 146 patients (34\%), cortical venous filling during ICA test occlusion was not synchronous. Nineteen of 42 patients (45\%) 


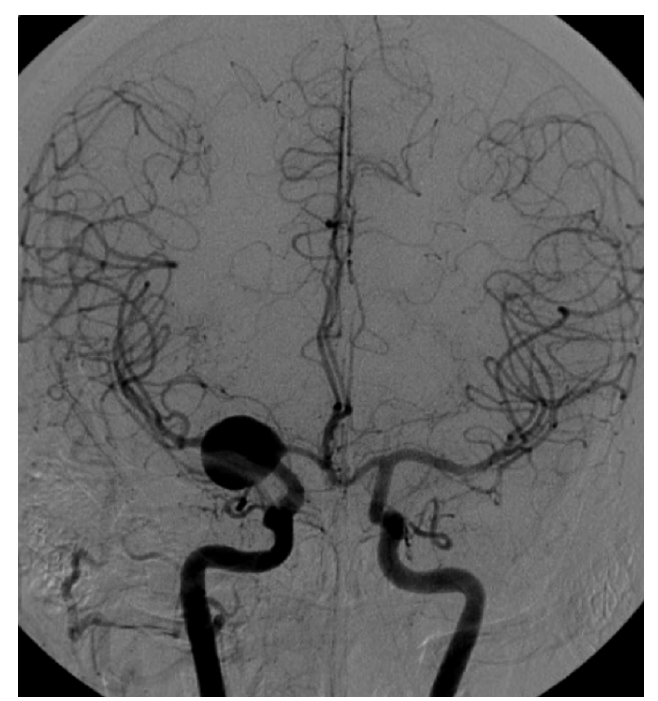

FIG 1. Large carotid tip aneurysm incidentally found in a 45-year-old woman. This aneurysm failed to thrombose after right ICA occlusion and was surgically treated 10 months later.

who were awake during test occlusion had neurologic symptoms. Twenty-one patients were treated with selective coiling, in 13 with stent or balloon assistance. Five patients had uncomplicated bypass surgery before ICA occlusion. One patient was treated with a flow diverter. One patient with severe head trauma and carotid cavernous fistula died soon after test occlusion. Twentytwo patients (with cavernous sinus aneurysms) were treated conservatively.

\section{Procedural and Postprocedural Complications}

There were no complications of the test occlusion in 146 patients ( $0 \%$ [ $95 \%$ CI, $0 \%-3 \%])$. Of 88 patients treated by ICA occlusion, $6(6.8 \%)$ had small hypoperfusion infarctions in the watershed zone between the anterior and middle cerebral artery on MR imaging 1-3 days after the occlusion. In 3 patients, this was subclinical; the other 3 patients had a mild transient hemiparesis. There were no thromboembolic ischemic complications. In another patient, a detachable balloon detached prematurely, and the empty balloon migrated with the blood flow into a left M3 branch. MR imaging after ICA occlusion demonstrated a localized ischemic area in the insula without clinical symptoms. There was 1 permanent neurologic complication in a 78-year-old woman who developed hypoperfusion infarction with hemiparesis as a result of hypovolemic shock due to a large retroperitoneal hematoma. The permanent complication rate was 1 of 88 (1.1\% [95\% CI, $1 \%-6.8 \%])$.

\section{Imaging Follow-Up}

Mean imaging and clinical follow-up was 35 months (median, 18 months; range, 3-180 months). Angiographic and MR imaging follow-up showed complete occlusion of 86 of 88 aneurysms $(97.7 \%)$ treated by ICA occlusion. In 2 patients (1.4\%), the aneurysm was not completely occluded on short-term follow-up. One patient with a supraclinoid ICA aneurysm had persistent filling of the aneurysm via the posterior communicating artery; this patient later underwent bypass surgery and clipping (Fig 1). The other patient had persistent filling of the giant carotid tip aneurysm at the 10-month follow-up angiogram, but MR imaging 3 months later demonstrated complete thrombosis (Fig 2).

At the latest MR imaging, 61 of 80 aneurysms (76\%) were decreased in size or were completely obliterated.

\section{Recovery of Cranial Nerve Function after ICA Occlusion}

Of the 62 patients who presented with cranial nerve dysfunction by mass effect of the aneurysm and were treated with ICA occlusion, 30 (48\%) were cured, 25 (40\%) improved, 6 (10\%) were unchanged, and 1 patient (2\%) was hemiplegic after a complication.

\section{DISCUSSION}

In this large cohort of consecutive patients with large and giant ICA aneurysms and therapeutic carotid artery occlusion after angiographic testing, the risk of permanent neurologic complications was extremely low. This study confirmed previous data from the same cohort and demonstrated that good clinical and anatomic results were sustained over time in a growing cohort of patients. These new data were more robust, with incremental narrowing of confidence intervals.

The only permanent neurologic complication was the result of hypovolemic shock due to an undetected retroperitoneal hematoma during ambulance transportation back to the referring hospital. Thus, the angiographic test occlusion as a predictor for tolerance to ICA occlusion proved to be very accurate, with no false-positives. The negative predictive value of the angiographic test occlusion could not be assessed because no ICA occlusion was performed when the test indicated nontolerance. Data from the era of surgical ICA clamp occlusion without previous tolerance testing indicate that approximately three-fourths of patients can tolerate ICA occlusion. ${ }^{16-18}$ In our cohort, the angiographic test indicated tolerance to permanent ICA occlusion in two-thirds of patients, which indicates that the proportion of false-negatives is probably limited.

After ICA occlusion, all aneurysms thrombosed completely except one, which makes the treatment very effective. The one exception was a large carotid tip aneurysm in a patient with a patent ipsilateral posterior communicating artery aneurysm that was not appreciated on angiography during test occlusion. Apparently, hemodynamic changes in the circle of Willis after ICA occlusion induced increased flow over the posterior communicating artery that prevented intraluminal thrombosis of the aneurysm. In aneurysms located distal to the posterior communicating artery, one should be aware of this phenomenon. We never encountered persistent aneurysm filling through collaterals via the ophthalmic artery. The effectiveness of our protocol of ICA balloon occlusion proximal to the aneurysm is comparable with the protocol of trapping the aneurysm with coils used by Labeyrie et al. ${ }^{19}$ However, we are in favor of our protocol because it is much easier to perform and definitely much cheaper. In addition, trapping in aneurysms located close to the ICA bifurcation is not possible because the distal ICA segment is too short to accommodate the coils.

During follow-up of at least 3 months in all the patients and much longer in most, three-fourths of the aneurysms decreased in size and almost $90 \%$ of the patients who presented with cranial 


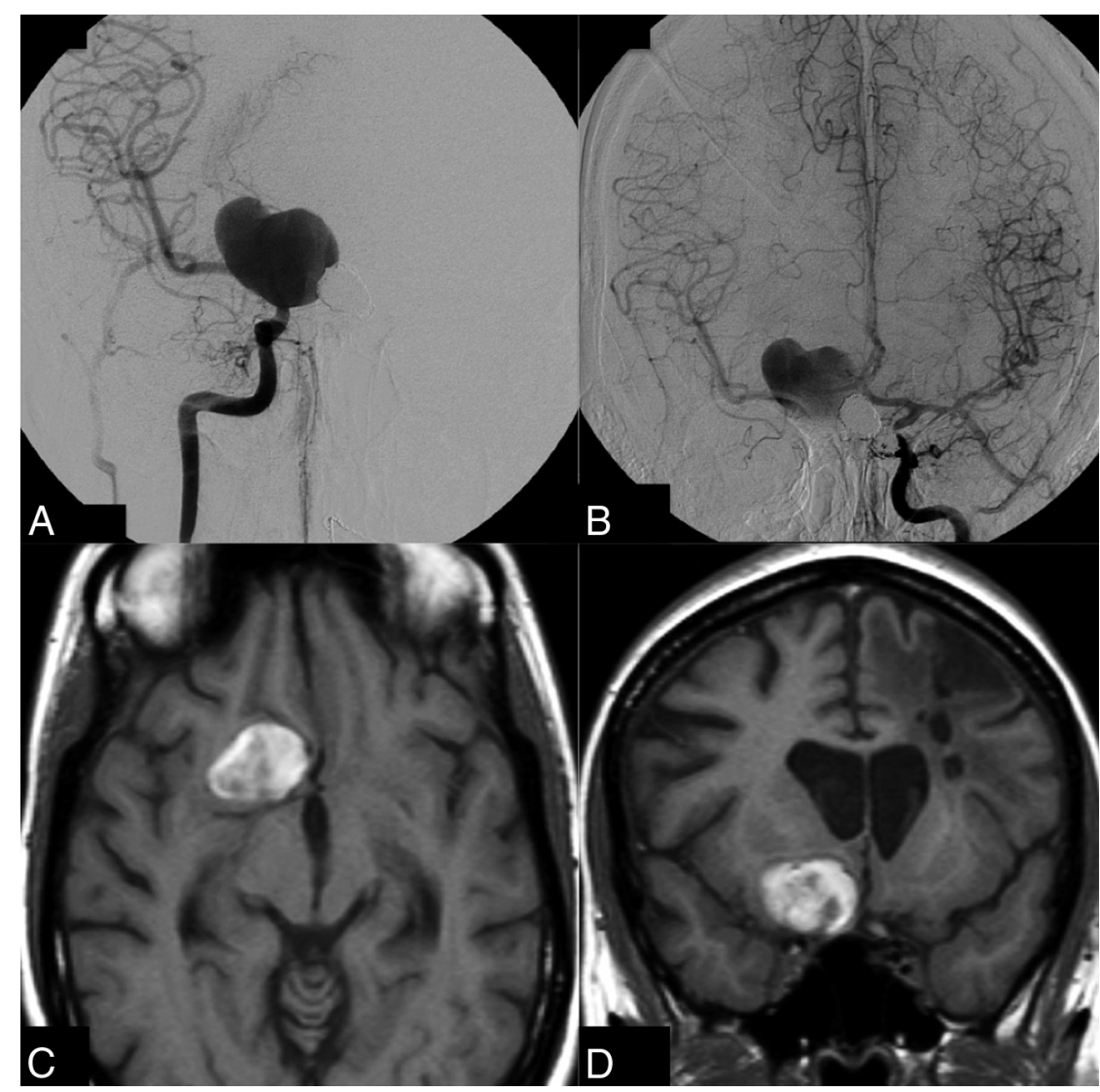

FIG 2. Giant unruptured right carotid tip aneurysm in a 36-year-old woman with multiple aneurysms. $A$, Right ICA angiogram shows the giant aneurysm; test occlusion showed ample collateral circulation via the anterior communicating artery. $B$, Left ICA angiogram 6 months after right ICA occlusion demonstrates that the aneurysm is still not occluded. $C$ and $D$, T1-weighted MR imaging 10 months after right ICA occlusion reveals complete thrombosis of the aneurysm lumen.

diverters for ICA aneurysms, also in patients who can tolerate ICA occlusion. ${ }^{22}$ However, recent meta-analyses that concern flow-diverter stents show that the combined morbidity and mortality rate is approximately $10 \%$, regardless of the type of aneurysm. ${ }^{23-29}$ Mortality was $4 \%$ in the study of Briganti et $\mathrm{al}^{24}$ in 76 patients with cavernous aneurysms treated with flow diverters. Many fatal complications with stent-assisted techniques are due to major hemorrhagic or ischemic complications, which are completely absent from carotid occlusion series. Because long-term imaging follow-up studies after flow-diverter treatment of ICA aneurysms are not yet available, complication rates may be underestimated: delayed in-stent occlusion may go clinically undetected in the three-fourths of patients who can tolerate ICA occlusion. ${ }^{30}$ In our opinion, supported by others, ${ }^{19}$ the safety profile of flow diverters is not good enough to justify treatment of ICA aneurysms in patients who can tolerate carotid occlusion, especially not in patients with cavernous aneurysms that generally exhibit a benign natural history.

ICA occlusion according to our protocol is also cost effective: angiographic test occlusion, followed by permanent ICA occlusion, is a straightforward pro-

nerve dysfunction were cured or improved. These favorable results on aneurysm size and symptoms of mass effect are in concordance with other studies. $^{7,20,21}$

Our data confirmed that ICA occlusion for large and giant carotid artery aneurysms was a very safe and effective therapy in patients with adequate collateral circulation. The angiographic test occlusion accurately predicted tolerance to ICA occlusion with probably only a few false-negatives but no false-positives. The angiographic test occlusion obviates the need for clinical testing and can be safely performed in patients under general anesthesia. After ICA occlusion and confirmation of aneurysm thrombosis on MR imaging, the aneurysm can be considered cured, and further imaging follow-up is not necessary. In a previous 3T MRA follow-up study in a selection of 26 patients of the present cohort, there were no de novo aneurysms after a mean follow-up of $>50$ months. In conjunction with other follow-up studies of patients with clipped aneurysms, there was no reason to believe that there is an increased risk of developing de novo aneurysms in patients with aneurysms treated by ICA occlusion compared with patients with clipped aneurysms. ${ }^{13}$ In another follow-up study by using MR arterial spin-labeling in 11 patients from this cohort after a mean 39-month follow-up, CBF values were within normal range and there was no significant CBF difference between hemispheres ipsilateral and contralateral to carotid sacrifice. ${ }^{15}$

Several investigators advocate ICA sparing therapy with flow cedure that takes 45-120 minutes. Occlusion balloons are cheap, and the patient is discharged home the next day. Prolonged imaging follow-up beyond the 3-month interval is usually not needed.

For patients with large and giant ICA aneurysms who cannot tolerate ICA occlusion, parent vessel sparing therapies should be considered with caution. In balancing the risks and benefits of these alternative therapies, it should be kept in mind that complications that lead to acute or delayed ICA occlusion in these patients certainly will cause neurologic deficit or even death. With the use of stents or flow diverters, this risk is not negligible: instent stenosis and occlusion occurs in approximately $10 \%$ of cases in the short term and the midterm. ${ }^{23}$ With bypass surgery, the risk of occlusion of the bypass is certainly present. ${ }^{4}$ The safest option to treat an aneurysm in a patient who cannot tolerate ICA occlusion seems to be selective coiling with or without balloon assistance. In patients with cavernous sinus aneurysms, conservative therapy can be the best option in a substantial proportion.

\section{CONCLUSIONS}

In patients with large and giant ICA aneurysms, ICA occlusion, when tolerated, remains a very safe and effective therapy. Tolerance to ICA occlusion can be reliably predicted by the angiographic test occlusion, also in patients under general anesthesia. In patients who cannot tolerate ICA occlusion, alternative treat- 
ments sparing the ICA should be offered only after careful benefit to risk analysis because complication rates of these therapies are substantially higher.

Disclosures: Charles Majoie-UNRELATED: Grants/Grants Pending: Dutch Heart Foundation*; Payment for Lectures (including service on speakers bureaus): Stryker.* *Money paid to the institution.

\section{REFERENCES}

1. van Rooij WJ. Endovascular treatment of cavernous sinus aneurysms. AJNR Am J Neuroradiol 2012;33:323-26 CrossRef Medline

2. van der Schaaf IC, Brilstra EH, Buskens E, et al. Endovascular treatment of aneurysms in the cavernous sinus: a systematic review on balloon occlusion of the parent vessel and embolization with coils. Stroke 2002;33:313-38 CrossRef Medline

3. van Rooij WJ, Sluzewski M, Beute GN. Ruptured cavernous sinus aneurysms causing carotid cavernous fistula: incidence, clinical presentation, treatment, and outcome. AJNR Am J Neuroradiol 2006;27:185-89 Medline

4. Brilstra EH, Rinkel GJ, Klijn CJ, et al. Excimer laser-assisted bypass in aneurysm treatment: short-term outcomes. J Neurosurg 2002;97: 1029-35 CrossRef Medline

5. Langer DJ, Van Der Zwan A, Vajkoczy P, et al. Excimer laser-assisted nonocclusive anastomosis: an emerging technology for use in the creation of intracranial-intracranial and extracranial-intracranial cerebral bypass. Neurosurg Focus 2008;24:E6 CrossRef Medline

6. van Rooij WJ, Sluzewski M. Endovascular treatment of large and giant aneurysms. AJNR Am J Neuroradiol 2009;30:12-18 CrossRef Medline

7. Lubicz B, Gauvrit JY, Leclerc X, et al. Giant aneurysms of the internal carotid artery: endovascular treatment and long-term follow-up. Neuroradiology 2003;45:650-55 CrossRef Medline

8. Lanzino G, Crobeddu E, Cloft HJ, et al. Efficacy and safety of flow diversion for paraclinoid aneurysms: a matched-pair analysis compared with standard endovascular approaches. AJNR Am J Neuroradiol 2012;33:2158-61 CrossRef Medline

9. D’Urso PI, Karadeli HH, Kallmes DF, et al. Coiling for paraclinoid aneurysms: time to make way for flow diverters? AJNR Am J Neuroradiol 2012;33:1470-74 CrossRef Medline

10. van Rooij WJ, Sluzewski M, Slob MJ, et al. Predictive value of angiographic testing for tolerance to therapeutic occlusion of the carotid artery. AJNR Am J Neuroradiol 2005;1:175-78 Medline

11. van Rooij WJ, Sluzewski M, Metz NH, et al. Carotid balloon occlusion for large and giant aneurysms: evaluation of a new test occlusion protocol. Neurosurgery 2000;47:116-21; discussion 122 CrossRef Medline

12. van Rooij WJ, Sluzewski M. Unruptured large and giant carotid artery aneurysms presenting with cranial nerve palsy: comparison of clinical recovery after selective aneurysm coiling and therapeutic carotid artery occlusion. AJNR Am J Neuroradiol 2008;29:997-1002 CrossRef Medline

13. de Gast AN, Sprengers ME, van Rooij WJ, et al. Long-term 3T MR angiography follow-up after therapeutic occlusion of the internal carotid artery to detect possible de novo aneurysm formation. AJNR Am J Neuroradiol 2007;28:508-10 Medline

14. de Gast AN, Sprengers ME, van Rooij WJ, et al. Midterm clinical and magnetic resonance imaging follow-up of large and giant carotid artery aneurysms after therapeutic carotid artery occlusion. Neurosurgery 2007;60:1025-29; discussion 1029-31 CrossRef Medline

15. Gevers S, Heijtel D, Ferns SP, et al. Cerebral perfusion long term after therapeutic occlusion of the internal carotid artery in patients who tolerated angiographic balloon test occlusion. AJNR Am J Neuroradiol 2012;33:329-35 CrossRef Medline

16. Linskey ME, Jungreis CA, Yonas $\mathrm{H}$, et al. Stroke risk after abrupt internal carotid artery sacrifice: accuracy of preoperative assessment with balloon test occlusion and stable xenon-enhanced CT. AJNR Am J Neuroradiol 1994;15:829-43 Medline

17. Drake CG, Peerless SJ, Ferguson GG. Hunterian proximal arterial occlusion for giant aneurysms of the carotid circulation. J Neurosurg 1994;81:656-65 CrossRef Medline

18. Fox AJ, Viñuela F, Pelz DM, et al. Use of detachable balloons for proximal artery occlusion in the treatment of unclippable cerebral aneurysms. J Neurosurg 1987;66:40 -46 CrossRef Medline

19. Labeyrie MA, Lenck S, Bresson D, et al. Parent artery occlusion in large, giant, or fusiform aneurysms of the carotid siphon: clinical and imaging results. AJNR Am J Neuroradiol 2015;36:140-45 CrossRef Medline

20. Abud DG, Spelle L, Piotin M, et al. Venous phase timing during balloon test occlusion as a criterion for permanent internal carotid artery sacrifice. AJNR Am J Neuroradiol 2005;26:2602-09 Medline

21. Zhang $\mathrm{Z}, \mathrm{Lv} \mathrm{X}, \mathrm{Wu} \mathrm{Z}$, et al. Clinical and angiographic outcome of endovascular and conservative treatment for giant cavernous carotid artery aneurysms. Interv Neuroradiol 2014;20:29-36 CrossRef Medline

22. Tanweer O, Raz E, Brunswick A, et al. Cavernous carotid aneurysms in the era of flow diversion: a need to revisit treatment paradigms. AJNR Am J Neuroradiol 2014;35:2334-40 CrossRef Medline

23. Velioglu M, Kizilkilic O, Selcuk $\mathrm{H}$, et al. Early and midterm results of complex cerebral aneurysms treated with Silk stent. Neuroradiology 2012;54:1355-65 CrossRef Medline

24. Briganti F, Napoli M, Tortora F, et al. Italian multicenter experience with flow-diverter devices for intracranial unruptured aneurysm treatment with periprocedural complications-a retrospective data analysis. Neuroradiology 2012;54:1145-52 CrossRef Medline

25. Berge J, Biondi A, Machi P, et al. Flow-diverter Silk stent for the treatment of intracranial aneurysms: 1-year follow-up in a multicenter study. AJNR Am J Neuroradiol 2012;33:1150-55 CrossRef Medline

26. Nelson PK, Sahlein D, Shapiro M, et al. Recent steps toward a reconstructive endovascular solution for the orphaned, complex-neck aneurysm. Neurosurgery 2006;59 (5 suppl 3):S77-92; discussion S3-13 CrossRef Medline

27. Kallmes DF, Hanel R, Lopes D, et al. International retrospective study of the Pipeline embolization device: a multicenter aneurysm treatment study. AJNR Am J Neuroradiol 2015;36:108-15 CrossRef Medline

28. Arrese I, Sarabia R, Pintado R, et al. Flow-diverter devices for intracranial aneurysms: systematic review and meta-analysis. Neurosurgery 2013;73:193-99; discussion 199-200 CrossRef Medline

29. Brinjikji W, Murad MH, Lanzino G, et al. Endovascular treatment of intracranial aneurysms with flow diverters: a meta-analysis. Stroke 2013;44:442-47 CrossRef Medline

30. Fiorella D, Hsu D, Woo HH, et al. Very late thrombosis of a Pipeline embolization device construct: case report. Neurosurgery 2010; 67(suppl operative):onsE313-34; discussion onsE314 CrossRef Medline 